ORIGINAL ARTICLE

\title{
Relationship of Recurrent Laryngeal Nerve with Inferior Thyroid Artery
}

\author{
Marina Saldanha ${ }^{1}$, Samatha K Jayaramaiah², Rajeshwary Aroor ${ }^{3}$, Vadisha S Bhat ${ }^{4}$, Sherin Varghese ${ }^{5}$
}

\begin{abstract}
Background: Recurrent laryngeal nerve (RLN) injury is a major concern in thyroid surgery as the effects can vary from hoarseness of voice to life-threatening complications like stridor. Hence, identification and preservation of the nerve is of utmost importance. The landmarks for identification are tracheoesophageal groove, inferior thyroid artery (ITA), ligament of berry, and tubercle of Zuckerkandl. The ITA is noted to be a constant landmark in identifying the RLN. The main aim of the study is to identify the relationship of the ITA with the RLN during thyroid surgery. Materials and methods: A total of 91 patients who underwent total thyroidectomy, completion, and hemithyroidectomy from January 2018 to December 2018 were included in the study. The position of the RLN with respect to the ITA was noted being as superficial, deep, or between the branches.

Results: Among a total of 150 RLNs, 140 (93.3\%) were identified and 10 were not identified. In 125 (89\%) dissections, the ITA was superficial to the RLN and in 15 dissections it was noted to be deep to the RLN.

Conclusion: Even though the RLN can be identified by various landmarks, the relationship with the ITA is consistent. However, as the nerve may be either superficial or deep to the ITA, knowledge of anatomy and variations of the RLN is necessary to ensure its preservation.

Clinical significance: The knowledge of relationship of the RLN with the ITA helps in identification and preservation of nerve, thus avoiding injury to the nerve and consequent complications.

Keywords: Inferior thyroid artery, Recurrent laryngeal nerve, Thyroidectomy.

Otorhinolaryngology Clinics: An International Journal (2019): 10.5005/jp-journals-10003-1318
\end{abstract}

\section{INTRODUCTION}

Thyroid surgeries are frequently performed endocrine surgical procedures worldwide. Recurrent laryngeal nerve (RLN) paralysis is one of the serious complications of thyroid surgery as it affects the individual's quality of life. The effects of unilateral RLN palsy vary from hoarseness of voice, aspiration, and can even become lifethreatening when bilateral. Hence, recognition and preservation of RLN during thyroid surgery is of utmost importance. The rate of RLN palsy worldwide ranges from $0.5 \%$ to $20 \%$ in thyroid surgeries. This depends on multiple factors like type of thyroid disease, surgical technique, anatomical variations, and surgeon's experience. ${ }^{1,2}$

Various studies have shown that identification of the RLN during surgery reduces the risk of injury of the nerve during dissection. ${ }^{3}$ During thyroid surgery, various landmarks help in identification of RLN such as the proximity of RLN to the inferior thyroid artery, its position in the tracheoesophageal groove, being identified lateral to the ligament of berry and it lies posterior to the tubercle of Zuckerkandl, when present. The course of RLN from the thorax to the larynx may have many variations in anatomy, which can make it vulnerable to injury during dissection. Hence, precise anatomical knowledge, identification of the landmarks, and knowledge of variations in its course are necessary to avoid injury to the nerve.

In various studies, it has been observed that the nerve enters the larynx after dividing into two or more branches. In dissection studies, the branching patterns of RLN were observed and it was noted that in $43 \%$ on both sides divided into one or more branches. ${ }^{4}$ From the inferior border of the cricoid cartilage, the distance of bifurcation or trifurcation ranges from $0.6 \mathrm{~cm}$ to $4.0 \mathrm{~cm}$. The extralaryngeal branches can arise at any point but it has been

\footnotetext{
${ }^{1-5}$ Department of Otorhinolaryngology, KS Hegde Medical Academy, NIITTE (Deemed to be University), Mangaluru, Karnataka, India

Corresponding Author: Marina Saldanha, Department of Otorhinolaryngology, KS Hegde Medical Academy, NIITTE (Deemed to be University), Mangaluru, Karnataka, India, Phone: +91 9663332251, e-mail: saldanhamarina@gmail.com
}

How to cite this article: Saldanha M, Jayaramaiah SK, Aroor R, et al. Relationship of Recurrent Laryngeal Nerve with Inferior Thyroid Artery. Int J Otorhinolaryngol Clin 2019;11(2):27-29.

Source of support: Nil

Conflict of interest: None

observed that below the level of the ITA branching is not common. ${ }^{5}$ Alteration in the normal anatomy due to neoplasm, goiter, or inflammatory changes can frequently vary the course of the RLN. In rare circumstances $(0.6 \%)$, the course of the right RLN does not turn around the subclavian artery but takes its origin directly from the vagus in the neck toward the larynx. This variation of the RLN is known as the "nonrecurrent" laryngeal nerve and is highly susceptible to surgical injury. ${ }^{6-8}$ The possibility of the nonrecurrent laryngeal nerve on the left side is rare but there are case reports of two clinical cases associated with a left retroesophageal subclavian artery and the right-sided aorta. ${ }^{9,10}$

The relation of RLN to the thyroid gland was found to be that, no case of the RLN penetrating the substance of the gland was observed. Based on the pathology, it was observed that attachment to the pretracheal fascia or its embedment in the ligament of Berry may increase the chances of nerve injury during surgery due to traction. ${ }^{10}$

The main aim of our study is to analyze the interrelationship of ITA with RLN during surgery of the thyroid. 


\section{Materials and Methods}

This prospective clinical study was conducted in the Department of Otorhinolaryngology, KS Hegde Medical Academy, between January 2018 and December 2018. Ethical clearance was obtained from the Institutional Ethics Committee.

In total, 91 patients underwent thyroidectomies of which 79 were females and 12 were males, with mean age of 46 years (range from 18 years to 80 years). A total of 49 patients underwent total thyroidectomy, 33 hemithyroidectomy, and 9 completion thyroidectomy. Computed tomography scan was done in cases of thyroid carcinoma and goiters extending to the mediastinum. All patients were subjected to videolaryngoscopy to see vocal cord movement preoperatively.

All thyroidectomies were done under general anesthesia by a team of experienced surgeons who followed a similar surgical technique. The standard technique of extracapsular dissection was followed. Important steps being ligation of the middle thyroid vein; during ligation of superior thyroid vessels care was taken to preserve the superior laryngeal nerve and blunt dissection of thyroid lobes to facilitate medial retraction. Identification of the ITA and the relationship between the ITA and RLN on both sides was noted as being superficial, deep, or between the branches of the artery. Once identified, the course of the nerve was followed from that point to its entry into the larynx and relation to other landmarks was noted.

\section{Results}

In 91 patients, RLN was identified in 140 (93.3\%) out of 150 dissections. The RLN was not identified in 10 dissections, which was 2 on the right side and 8 on the left. The ITA was found superficial to the nerve in 125 (89\%) dissections and deep to the nerve in 15 (11\%). Table 1 summarizes the histopathological diagnosis of the patients who underwent thyroidectomy. The interrelationship between the RLN and the ITA on both sides is shown in Table 2 .

In our study, we noted extralaryngeal branching in two dissections on the right side. The bifurcation of the RLN was above the level of ITA in both the cases. We did not observe the nerve coursing between the branches of ITA as well as the presence of the nonrecurrent laryngeal nerve in our dissections.

Table 1: Thyroid diseases

\begin{tabular}{lrr}
\hline Histopathological diagnosis & $n$ & $\%$ \\
\hline Nodular colloid goiter & 59 & 65 \\
Follicular adenoma & 9 & 10 \\
Hashimoto's thyroiditis & 7 & 8 \\
Hürthle cell adenoma & 5 & 5 \\
Papillary carcinoma & 7 & 8 \\
Follicular carcinoma & 2 & 2 \\
Oncocytic adenoma & 1 & 1 \\
Lymphocytic thyroiditis & 1 & 1 \\
\hline
\end{tabular}

Table 2: Relationship between recurrent laryngeal nerve and inferior thyroid artery on both sides

\begin{tabular}{|c|c|c|c|c|}
\hline \multirow{2}{*}{$\begin{array}{l}\text { Course of inferior } \\
\text { thyroid artery }\end{array}$} & \multicolumn{2}{|c|}{ Right } & \multicolumn{2}{|c|}{ Left } \\
\hline & No. & $\%$ & No. & $\%$ \\
\hline Superficial to nerve & 74 & 95 & 51 & 82 \\
\hline Deep to nerve & 4 & 5 & 11 & 18 \\
\hline Total & 78 & 100 & 62 & 100 \\
\hline
\end{tabular}

Identification of the RLN was challenging in two cases of Hashimoto's thyroiditis as there were adhesions to the trachea. Difficulty in identification due to adhesions was also noted in two cases of papillary carcinoma of thyroid and three case of huge multinodular goiter.

\section{Discussion}

Identification and preservation of RLN is vital in thyroid surgeries. A thorough knowledge of anatomy of the nerve and also its variations in the course avoids injury during dissection. It is better to carefully search for the nerve during dissection as avoidance of the nerve during dissection carries a high risk (10\%) of damaging the nerve permanently. ${ }^{10}$ The ITA is used as a reliable landmark to identify the nerve. It divides into many branches that may pass either superficially, deep, or among the branches of the nerve. However, the anatomical relationship between the two structures is not constant on both sides. ${ }^{11}$

In our study, we observed that ITA was superficial to RLN in 125 (89\%) dissections and deep in 15 (11\%) intraoperatively. On the right side, ITA was superficial to RLN in 95\% and deep in 5\%, while on the left side ITA was superficial to RLN in $82 \%$ and deep in $18 \%$.

A similar observation was made by Ardito et al. ${ }^{12}$ where $61 \%$ of the cases had ITA superficial to RLN, $12 \%$ were deep, and $27 \%$ between the branches on the right side while on the left side ITA was superficial to RLN in $77 \%$, deep in $1.9 \%$, and $27 \%$ between the branches. In a study conducted by Hisham and Lukman ${ }^{11}$ observation was that RLN was passing posterior to or in between branches of the ITA in $83.8 \%$ and anterior to ITA in $16.2 \%$. In contrast to our study, Sturniolo et al. ${ }^{13}$ noticed that $22 \%$ had ITA superficial to RLN on the right side, while $36 \%$ had deep to RLN on the left. Steinberg et al., ${ }^{14}$ reported that the RLN traverses between the branches of the ITA in about $6.5 \%$, deep in $61.5 \%$, and superficial in $32.5 \%$. The general consensus based on different series suggests that the authors have accepted that the RLN most commonly lies deep to the ITA.

In our study, extralaryngeal branching was observed in two dissections on the right side. In both cases, bifurcation of the RLN was seen above the level of ITA.

Hisham and Lukman ${ }^{11}$ in his study observed the extralaryngeal branching of the nerve. Galen's anastomosis is formed by the posterior branch of the RLN and the internal laryngeal branch of the superior laryngeal nerve. However, the branching pattern was found to be inconsistent and variable. This nerve is not commonly encountered but it is vulnerable to injury, and meticulous dissection will avoid inadvertent injury.

The experience of the surgeon in dissection of RLN, as opposed to an inexperienced one, is known to account for the differences in the frequency of vocal cord paralysis. Other contributory factors to nerve injury are dissecting or stripping the nerve of its ensheathing fascia, using bipolar cautery at the site of entry of the RLN into the cricoarytenoid joint area or excessive traction on the gland. When the nerve has not been identified inferiorly, a search for it in the upper part of the gland should be made by identifying the ligament of Berry or the inferior cornu of the thyroid cartilage. If RLN is not found in its normal position, the possibility of the nonrecurrent nerve should be kept in mind. ${ }^{10}$

In our dissections, we observed that on the left side of the neck, the course of the RLN in the tracheoesophageal groove was closest at the inferior end. On the right, the RLN reaches the middle third of the groove with an oblique course. In addition, Hunt ${ }^{10}$ observed in his study that the RLN is located in the tracheoesophageal groove 
in $65 \%$ on the right and $77 \%$ on the left. Hence, it can be used as one of the reliable landmarks for identifying the RLN.

\section{Conclusion}

In literature, various techniques have been described to locate the course of the RLN during surgery. A thorough knowledge of normal anatomy and variations in the course of the nerve is essential in order to ensure the integrity and safety of the RLN in thyroid surgery. We conclude that the relationship of RLN with ITA is consistent and can be used during thyroid surgery as a reliable landmark in identification of the nerve.

\section{Clinical Significance}

The knowledge of the anatomical relationship of RLN with ITA helps in identification of the nerve during thyroid surgery thus avoiding injury to the nerve and consequent complications.

\section{References}

1. Calo PG, Pisano G, Medas F, et al. Identification alone versus intraoperative neuromonitoring of the recurrent laryngeal nerve during thyroid surgery: experience of 2034 consecutive patients. J Otolaryngol Head Neck Surg 2014;43(1):16. DOI: 10.1186/1916-0216-43-16.

2. Dralle $\mathrm{H}$, Sekulla $\mathrm{C}$, Lorenz $\mathrm{K}$, et al. Intra-operative monitoring of recurrent laryngeal nerve in thyroid surgery. World J Surg 2008;32(7):1358-1366. DOI: 10.1007/s00268-008-9483-2.

3. Chiang FY, Lu IC, Chen HC, et al. Intraoperative neuromonitoring for early localization and identification of recurrent laryngeal nerve during thyroid surgery. Kaohsiung J Med Sci 2010;26(12):633-639. DOI: 10.1016/S1607-551X(10)70097-8.
4. Rustad WH, Morrison LE. Revised anatomy of the recurrent laryngeal nerves: surgical importance based on the dissection of 100 cadavers. Laryngoscope 1952;62(3):237-249. DOI: 10.1288/00005537195203000-00001.

5. Nemiroff PM, Katz AD. Extralaryngeal branches of recurrent laryngeal nerve: surgical and clinical significance. Am J Surg 1982;144(4): 466-469. DOI: 10.1016/0002-9610(82)90425-1.

6. Proye CA, Carnaille BM, Goropouls A. Nonrecurrent and recurrent laryngeal nerve: a surgical pitfall in cervical exploration. Am J Surg 1991;162:495-496. DOI: 10.1016/0002-9610(91)90271-E.

7. Stewart GL, Mountain GC, Colcock BP. Nonrecurrent laryngeal nerve. Br J Surg 1972;59(5):379-381. DOI: 10.1002/bjs.1800590513.

8. Henry JF, Audiffret J, Denizot A, et al. The nonrecurrent inferior laryngeal nerve: review of 33 cases including two on the left side. Surgery 1988;104(6):977-984.

9. Berlin DD. The recurrent laryngeal nerves in total ablation of the normal thyroid gland: an anatomical and surgical study. Surg Gynecol Obstet 1935;60:19-26.

10. Hunt PS. A reappraisal of the surgical anatomy of thyroid and parathyroid glands. Br J Surg 1968;55(1):63. DOI: 10.1002/ bjs.1800550119.

11. Hisham AN, Lukman MR. Recurrent laryngeal nerve in thyroid surgery: a critical appraisal. ANZ J Surg 2002;72(12):887-889. DOI: 10.1046/j.1445-2197.2002.02578.x.

12. Ardito G, Revelli L, D'Alatri L, et al. Revisited anatomy of the recurrent laryngeal nerves. Am J Surg 2004;187(2):249-253. DOI: 10.1016/ j.amjsurg.2003.11.001.

13. Sturniolo G, D'Alia C, Tonante A, et al. The Recurrent Laryngeal Nerve Related to Thyroid Surgery. Am J Surg 1999;177(6):485-488. DOI: 10.1016/S0002-9610(99)00101-4.

14. Steinberg JL, Khane GJ, Fernandez CMC, et al. Anatomy of the recurrent laryngeal nerve: a redescription. J Laryngol Otol 1986;100(8):919-927. DOI: 10.1017/S0022215100100325. 